\title{
EIT Four-Level Lambda Scheme of Cold Rubidium Atoms
}

\author{
B. Brzostowski ${ }^{1}$, V. Cao Long ${ }^{1}$, K. Dinh Xuan ${ }^{2}$, B. Grabiec ${ }^{1}$, \\ S. Vu $\operatorname{Ngoc}^{2}$, A. $\dot{Z}^{2} a b a^{1}$ \\ ${ }^{1}$ Quantum Optics and Engineering Division, Institute of Physics, \\ University of Zielona Góra, ul. Prof. Szafrana 4a, 65-516 Zielona Góra, Poland \\ e-mail:B.Brzostowski@proton.if.uz.zgora.pl \\ ${ }^{2}$ Vinh University, Nghe An, Vietnam
}

(Received: 29 June 2010; accepted: 13 September 2010)

\begin{abstract}
In this paper, using the general master equation in the dipole interaction and the rotating-wave approximation, we will explain the experimental results obtained by Warsaw Group for Electromagnetically Induced Transparency in four-level lambda scheme of cold rubidium atoms. The theoretical calculations are in good agreement with the experimental data.
\end{abstract}

Key words: electromagnetically induced ransparency (EIT), cold Rb atoms in MOT, lambda scheme

\section{INTRODUCTION}

Electromagnetically induced transparency (EIT) [1] is a quantum interference effect that permits propagation of light through an opaque atomic medium without attenuation. Early experimental studies of EIT were mainly carried out in rubidium atoms and concerned with three-level systems (mainly systems), which are elementary systems for studying EIT. They could form lambda, vee or cascade configurations [13]. EIT manifests itself as a narrow window of transparency within the absorption profile and it is accompanied by a step, positive dispersion of the refractive index. It was first proposed theoretically in 1989 [2] and experimentally verified in 1991 [3]. Since then, theoretical and experimental studies of EIT have attracted great attention due to their potential applications in many fields, such as low light nonlinear optics [4], quantum information [5], atomic frequency standard [6], physics of semiconductor quantum wells and quantum dots [14-21], photonic crystals [22] and so on. Several excellent reviews on the progress in EIT and other quantum coherence phenomena are available [23-29] giving a deeper insight into the subject and providing lists of original references.

Recently, many groups explored the EIT phenomena using the laser cooled atoms. The cold atoms are confined in a magneto-optical trap (MOT) [7]. There are several advantages in the cold atoms [8]. Firstly, because of the low temperature of the cold atoms (below $\mathrm{mK}$ ), the Doppler broadening effect is effectively eliminated. This allows the coupling and the probe beams to propagate in arbitrary directions and to have various kinds of polarizations configurations in the experiments of studying the quantum coherence phenomena. The flexibility of the experimental arrangements and the degree of freedom of the studies are improved. Secondly, the motion of cold atoms is very slow. It follows that we could have a very high density sample of cold atoms for the studies and the collision perturbation is still negligible. The lower collision rates in the cold atomic sample reduce greatly the decoherence rate. The recent studies on EIT and the related phenomena in the cold atoms provided intensive understanding of the atomic coherence and interference in the fundamental interaction between the light field and the atoms.

Some studies of EIT in the cascade configuration have been made before [9], but when the degeneration of the Zeeman levels are taken into account, we have the multilevel cascade systems. Although essential physics about EIT has been understood well from the studies of the simple three-level systems, there are several interesting 
features in the complicated multi-level systems [10]. In their paper Wang and his coworkers have reported their experimental study of EIT in a multi-level cascade system in cold ${ }^{85} \mathrm{Rb}$ atoms. It has been shown that the experimental measurements agree well with the theoretical calculations.

Recently, the Warsaw Group of Physicists have performed a similar experiment and observed a resonance of enhanced transparency (transparency window) in the absorption profile of a weak probe beam (p) in the presence of a strong beam (c) beam. The probe and coupling beams were approximately at $60^{\circ}$ and with orthogonal polarizations. In our previous papers [30, 31], we have calculated theoretically the EIT spectra using the master equation introduced in [10] for a five-level model approximation. Our theoretical results are in good agreement with the experimental data obtained by Warsaw Group.

In this paper we will use a four-level scheme for the lambda configuration for describing other experimental observations performed also by Warsaw Group [32]. Obtained results are in good agreement again with the experimental data.

\section{MASTER EQUATION FOR THE FOUR-LEVEL EIT SYSTEM}

A 4-level model of the electromagnetically induced transparency (EIT) in $\Lambda$-configuration was considered. The states in Fig. 1 correspond to the following atomic states

$$
\begin{aligned}
& |1\rangle=5 S_{1 / 2} \quad(F=2),|2\rangle=5 S_{1 / 2} \quad\left(F^{\prime}=2\right), \\
& |3\rangle=5 P_{1 / 2} \quad(F=3),|4\rangle=5 P_{1 / 2} \quad\left(F^{\prime}=3\right) .
\end{aligned}
$$

The strong beam $\omega_{c}$ couple the state $|1\rangle$ with states $|3\rangle$ and $|4\rangle$, while the weak probe beam $\omega_{p}$ brings about a transitions $|2\rangle$ to $|3\rangle$ and $\Delta_{1}=\omega_{p}-\omega_{32}, \Delta_{2}=\omega_{c}-\omega_{31}$ are detunings. Where $\omega_{32}$ and $\omega_{31}$ are resonance frequencies, while $\Omega_{p}=\mu_{32} E_{p} / \hbar$ and $\Omega_{c}=\mu_{31} E_{c} / \hbar$ are Rabi frequencies $\left(\delta_{1}=\omega_{21}, \delta_{2}=\omega_{43}\right)$.

The evolution of the atomic variables in the interaction and RWA approximation is governed by the master equation

$$
\begin{aligned}
\frac{\partial \rho}{\partial t} & =-\frac{i}{\hbar}[H, \rho]+\gamma_{21} L_{21} \rho+\gamma_{32} L_{32} \rho+ \\
& +\gamma_{41} L_{41} \rho+\gamma_{42} L_{42} \rho+\gamma_{43} L_{43} \rho .
\end{aligned}
$$

The total Hamiltonian of 4-level system can be written as

$$
H=H_{0}+H_{\mathrm{I}}
$$

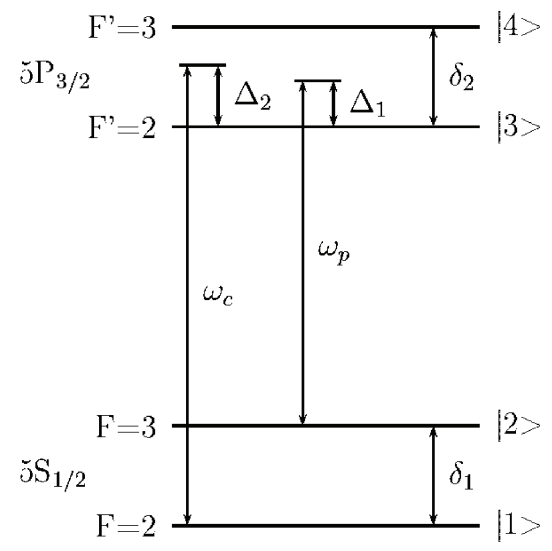

Fig. 1. The four-level scheme

Here $H_{0}$ is the unperturbed Hamiltonian

$$
\begin{gathered}
H_{0}=\hbar\left(\Delta_{1}-\Delta_{2}\right) \sigma_{22}-\hbar \Delta_{2} \sigma_{33}+ \\
+\hbar\left(\delta_{2}-\Delta_{2}\right) \sigma_{44}
\end{gathered}
$$

and $H_{\mathrm{I}}$ is the atom-field interaction Hamiltonian

$$
\begin{gathered}
H_{\mathrm{I}}=-\hbar \Omega_{c}\left(\sigma_{13}+\sigma_{31}\right)-\hbar a_{41} \Omega_{c}\left(\sigma_{14}+\sigma_{41}\right)+ \\
-\hbar \Omega_{p}\left(\sigma_{32}+\sigma_{23}\right)-\hbar b_{42} \Omega_{p}\left(\sigma_{42}+\sigma_{24}\right) .
\end{gathered}
$$

The Hamiltonians $H_{0}$ and $H_{\mathrm{I}}$ in Eqs. (3)-(5) are expressed in the basis of selected states where $\sigma_{i j}=|i\rangle\langle j|$ $(i, j=1,2,3,4)$ are population-operator for $i=j$, and dipole operator for $i \neq j$. Decay of an atom in Eq. (2) from level $i$ to level $j$ is described by the formulae

$$
L_{i j} \rho=\frac{1}{2}\left(2 \sigma_{j i} \rho \sigma_{i j}-\sigma_{i j} \sigma_{j i} \rho-\rho \sigma_{i j} \sigma_{j i}\right) .
$$

Parameters $a_{41}=\mu_{41} / \mu_{31}, \quad b_{42}=\mu_{42} / \mu_{32} . \quad \gamma_{i j}$ is the rate spontaneous emission for transition from level $i$ to level $j$.

The probe absorption signal can be obtained from the density matrix Eq. (2) by solving them numerically or analytically under the steady state condition, i.e.

$$
\frac{\partial \rho}{\partial t}=0
$$

We numerically solve the rate equations in the steady state regime for different values of pump and probe detunings. The probe absorption signal is proportional to the imaginary part of the $\rho_{2 j}(j=3,4)$. We can write it as:

$$
\alpha\left(\omega_{p}\right)=\sum_{j=3}^{4} \operatorname{Im}\left(\rho_{2 j}\right) .
$$




\section{COMPARISON WITH THE EXPERIMENTAL DATA OF WARSAW GROUP}

In the experiment of Warsaw Group [12] the temperature of atom cloud is about $100 \mu \mathrm{K}$. The diameter of coupling beam is approximately equal to $2 \mathrm{~mm}$, its power is $400 \mathrm{~mW}$. The weak probe beam has the diameter $0.7 \mathrm{~mm}$ and the power $100 \mathrm{~mW}$.

Our results for the transition $5 S_{1 / 2}(F=3) \rightarrow 5 P_{3 / 2}(F=3)$ are compared with experimental data in Fig. 2 and Fig. 3. The experimental data are denoted by solid lines and the dashed lines correspond to the our numerical calculations. In Fig. 2, detuning of the coupling beam in the experiment is around $150 \mathrm{MHz}$, whereas in Fig. 3 it is approximately $90 \mathrm{MHz}$.

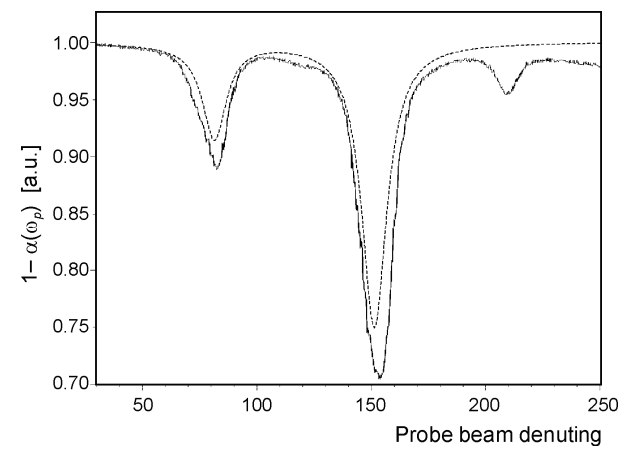

Fig. 2. Transmission rate as the function of probe beam detuning. Solid line corresponds to the experimental results of Warsaw Group with coupling beam detuning approximately equal to $150 \mathrm{MHz}$. Theoretical results are plotted as dashed line (for the parameters see in the text)

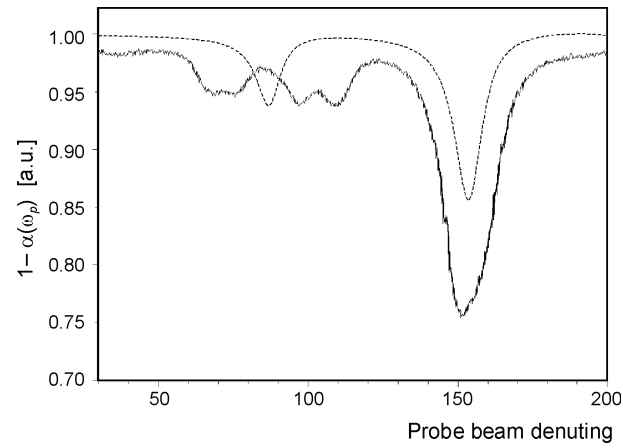

Fig. 3. The same as in Fig. 2 but for experimental results corresponding to the coupling beam detuning approximately equal to $90 \mathrm{MHz}$. Values of parameters involved in the theoretical problem are given in the text

In Fig. 2, we have the following values of parameters involved in our model: $a_{41}=0.9, b_{42}=1, \delta_{2}=70 \mathrm{MHz}$, $\Omega_{c}=34 \mathrm{MHz}, \Omega_{p}=3 \mathrm{MHz}, \Delta_{2}=150 \mathrm{MHz}, \gamma_{31}=\gamma_{32}=1 \mathrm{MHz}$, $\gamma_{41}=0.5 \mathrm{MHz}$ and $\gamma_{42}=10 \mathrm{MHz}$, whereas in Fig. 3: $\Delta_{2}=90 \mathrm{MHz}, \gamma_{31}=0.5 \mathrm{MHz}, \gamma_{32}=0.25 \mathrm{MHz}, \gamma_{41}=3 \mathrm{MHz}$, $\gamma_{42}=10 \mathrm{MHz}$ and other parameters are the same as before. It follows that the calculations based on the master equation generally agree with the experimental results. However, the model proposed in this paper should be extended to the case when additional Zeeman sublevels will be taken into account. This is the subject of our further publications.

We would like to express our deep gratitude to the Warsaw Experimental Group of Polish Academy of Science for giving us their experimental data before publications.

\section{References}

[1] M.D. Lukin, A. Imamoglu, Nature 413, 273 (2001).

[2] A. Imamoglu, S.E. Harris, Opt. Lett. 14, 1344 (1989).

[3] K.J. Boller, A. Imamoglu, S.E. Harris, Phys. Rev. Lett. 66, 2593 (1991).

[4] S.E. Harris, L.V. Hau, Phys. Rev. Lett. 82, 4611 (1999).

[5] M. Paternostro, M.S. Kim, B.S. Ham, Phys. Rev. A67, 023811 (2003).

[6] S. Knappe, J. Kitching, L. Hollberg, Appl. Phys. Lett. 81, 553 (2002).

[7] Cao Long Van, Dinh Xuan Khoa, Marek Trippenbach, Introduction to Nonlinear Optics, Vinh 2003.

[8] Y.C. Chen, C.W. Lin, I.A. Yu, Phys. Rev. A61, 053805, (2000).

[9] J. Clarke, H.X. Chen, W.A. van Wijngaarden, Appl. Opt. 40, 2047 (2001)

[10] J. Wang et al., Phys. Lett. A328, 437 (2004).

[11] M.S. Safronova, C.J. Williams, C.W. Clark, Phys. Rev. A69, 022509 (2004).

[12] A.M. Glodz et al., to be published and K. Kowalski, Elektromagnetycznie wymuszona przezroczystość w zimnych atomach rubidu $w$ pulapce magneto-optycznej, $\mathrm{PhD}$ thesis (in polish), Institute of Physics, Polish Academy of Sciences, Warsaw 2008

[13] J.R. Boon, E. Zekou, D. McCloin, M.H. Dunn, Phys. Rev. A59, 4675 (1999).

[14] M. Phillips, H. Wang, Opt. Lett. 28, 831 (2003).

[15] G.B. Serapiglia et al., Phys. Rev. Lett. 84, 1019 (2000).

[16] M.C. Phillips, H. Wang, Phys. Rev. Lett. 89, 186401 (2002).

[17] W.W. Chow, H.C. Schneider, M.C. Phillips, Phys. Rev. A68, 053802 (2003).

[18] M.C. Phillips et al., Phys. Rev. Lett. 91, 183602 (2003).

[19] J. Houmark et al. J. Phys. Conf. Ser. 107, 012005 (2008).

[20] Y. Wu, X. Yang, Phys. Rev. A71, 053806 (2005).

[21] S. Marcinkievicius, A. Gushterov, J.P. Reithmaier, Appl. Phys. Lett. 92, 04113 (2008).

[22] H. Gersen, Phys. Rev. Lett. 94, 073903 (2005).

[23] E. Arimondo, Prog. Opt. 35, 257 (1996).

[24] S.E. Harris, Phys. Today 50, 36 (1997).

[25] J.P. Marangos, J. Mod. Opt 45, 471 (1998).

[26] R.W. Boyd, D.J. Gauthier, Prog. Opt. 43, 497 (2002).

[27] M.D. Lukin, Rev. Mod. Phys. 75, 457 (2003).

[28] M. Fleischhauer, A. Imamoglu, J.P. Marangos, Rev. Mod. Phys. 77, 633 (2005).

[29] A. Andre et al., J. Phys. B38, 5589 (2005).

[30] V. Cao Long, K. Dinh Xuan, T. Bui Dinh, H. Nguyen Viet, Comm. in Physics 18, 146 (2008).

[31] K. Kowalski et al., J. of Non-Crystalline Solids, 355, 1295 (2009). 

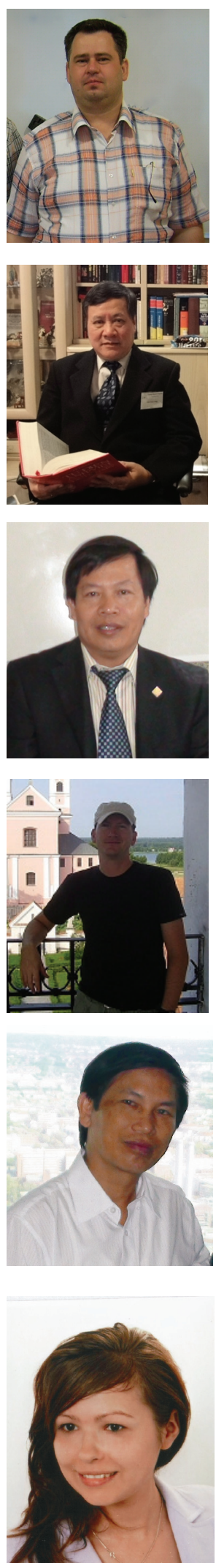

Bartosz BrzostowsKI works at the Institute of Physics of the University of Zielona Góra, Poland. He received Ph.D. degree in Physics in 2003 from University of Wrocław. His main areas of interest include quantum optics, solid state physics and several topics in molecular physics (mainly magnetic molecules).

CAO LONG VAN was born in 1952 in Hanoi, Vietnam. He graduated from Warsaw University in 1976 with an MSc degree in Theoretical Physics. His master thesis was entitled "Callan-Symanzik Equation and Transition Probabilities" and was written under the supervision of Prof. Iwo Białynicki-Birula. In 1979, he defended his PhD dissertation entitled "Phase Representation in Quantum Optics", also supervised by Prof. I. Białynicki-Birula. He habilitated in 1987 in Center of Theoretical Physics, Polish Academy of Sciences in Warsaw with a thesis entitled "Noises in Quantum Optics". His research interests concern several topics in Quantum and Nonlinear Optics.

DinH XuAn KhOA graduated from Vinh University, Vietnam, with a major in physics in 1981. He completed a PhD course in Quantum Optics in 1996. His doctoral thesis entitled "Generative Kinetics of Dye Lasers" was supervised by Prof. Cao Long Van and Prof. Dao Xuan Hoi. His field of interests covers a large variety of topics in Quantum and Nonlinear Optics. He is exceptionally interested in Soliton Theory and research concerning long-distance optical communication systems. Recently, Prof. Dinh Xuan Khoa has been a deputy rector of Vinh University.

Bogdan Grabiec was born in Jastrzębie Zdrój, Poland. He received the Ph.D. degree in Physics in 2002 from University of Silesia. He is working at the Institute of Physics, the University of Zielona Góra. His research interests concern quantum optics and physics solids state.

SAU VU NGOC was born in Thanh Hoa province, Vietnam. He graduated, with a major in Physics, from Vinh University in 1976. He completed a PhD course in Quantum Optics in 1996. His doctoral thesis entitled "Two-photon pumped Dye Lasers". Since 2001 he has been selected as the dean of faculty of technology. His field of interests covers a large variety of topics in Quantum and Nonlinear Optics, Laser physics, Atomic and molecular Physics.

AgNIESZKa ŻABA was born in 1985 Szprotawa, Poland. She graduated from the University of Zielona Góra in 2009 with an MSc degree in Physics. Her master thesis was entitled „Influence of hiperfine structure on electromagnetically induced transparency in cold rubidium atoms" and was written under the supervision of Prof. Cao Long Van. She is interested in quantum optics. 